\title{
Efeitos de um programa de Mat Pilates sobre indicadores de saúde em servidores de um hospital
}

\section{Effects of a Mat Pilates program on health indicators in servers of a hospital}

\author{
Carla Borges Feldner ${ }^{1}$, Nathallie de Freitas Cezário², Edmar Lacerda Mendes ${ }^{3}$
}

http://dx.doi.org/10.11606/issn.2238-6149.v30i3p160-166

Feldner CB, Cezário NF, Mendes EL. Efeitos de um programa de Mat Pilates sobre indicadores de saúde em servidores de um hospital. Rev Ter Ocup Univ São Paulo. 2020 set.-dez.;30(3):160-6.

RESUMO: Objetivo: Avaliar o efeito de um programa Mat Pilates sobre a composição corporal, qualidade de vida, nível de atividade física, flexibilidade e força em servidores de um hospital universitário. Procedimentos metodológicos: Trata-se de um estudo quasiexperimental com 22 servidores, que participaram de um programa de Mat Pilates uma vez por semana, durante 20 semanas. Os servidores foram submetidos à aferição da pressão arterial, avaliação corporal, teste de força abdominal e flexibilidade, e preencheram questionários de qualidade de vida, saúde e nível de atividade física, antes e depois da intervenção. Para análise das variáveis contínuas e categóricas foram utilizados os testes t pareado e qui-quadrado, respectivamente, ao nível de significância $p<0,05$. Resultados: Após intervenção observouse redução significativa do perímetro da cintura, pressão arterial sistólica e diastólica, massa corporal gorda e percentagem de gordura. Ainda, houve melhora dos níveis de atividade física, força abdominal, flexibilidade e domínios da qualidade de vida. Conclusão: Mat Pilates se mostra eficiente em melhorar parâmetros hemodinâmicos, físicos e de qualidade de vida em servidores de um hospital universitário.

PALAVRAS-CHAVE: Qualidade de vida; Exercício físico; Saúde do trabalhador.
Feldner CB, Cezário NF, Mendes EL.Effects of a Mat Pilates program on health indicators in servers of a hospital. Rev Ter Ocup Univ São Paulo. 2020 Sept.-Dec.;30(3):160-6.

\begin{abstract}
Objective: To evaluate the effect of a Mat Pilates program regarding the body composition, quality of life, level of physical activity, flexibility and strength in servants of a university hospital. Methodological procedures: This is a quasi-experimental study with 22 servers, who participated in a Mat Pilates program once a week for 20 weeks. The servers were submitted to blood pressure measurement, body evaluation, abdominal strength test and flexibility, and filled out questionnaires on quality of life, health and physical activity level, before and after the intervention. For the analysis of the continuous and categorical variables, paired and chi-square tests were used, respectively, at the significance level $\mathrm{p}<0.05$. Results: A significant reduction in waist circumference, systolic and diastolic blood pressure, fat body mass and fat percentage were observed after intervention. Still, there was improvement of physical activity levels, abdominal strength, flexibility and domains of quality of life. Conclusion: Mat Pilates is efficient in improving hemodynamic, physical and quality of life parameters in servants of a university hospital.
\end{abstract}

KEYWORDS: Quality de life; Exercise; Occupational health.

Este artigo é integrante de um projeto de extensão denominado Mat Pilates para servidores da Universidade Federal do Triângulo Mineiro.

1. Residente Multiprofissional em Saúde do Adulto pela Universidade Federal do Triângulo Mineiro - UFTM e Graduada em Educação Física pela Universidade Federal de Uberlândia. https://orcid.org/0000-0003-3348-4104 E-mail: carlafeldner@hotmail.com (Feldner CB)

2. Mestre em Ciências da Saúde pela Universidade Federal de Uberlândia (UFU), Residência Multiprofissional em Saúde/UFU e Graduada em Educação Física/UFU. https://orcid.org/0000-0003-3468-0174 E-mail: nathalliecezario@gmail.com (Cezário NF)

3. Departamento de Ciências do Esporte, Programa de Pós-Graduação em Educação Física, Grupo de Pesquisa em Ciência do Exercício, Saúde e Performance Humana/UFTM, Doutor em Biologia Celular e Estrutural pela Universidade Federal de Viçosa. https://orcid.org/0000-0001-8182-8296 E-mail: edmar.mendes@uftm.edu.br (Mendes EL)

Endereço de correspondência: Nathallie de Freitas Cezário. Rua Antônio Borges Araújo, 465, Mercês, Uberaba-MG 


\section{INTRODUÇÃO}

$\mathrm{O}^{-}$ trabalhadores possuem características próprias no seu ambiente de trabalho, muitas vezes passam horas em posição estática na postura sentada ou em pé a exercer atividades repetitivas. Assim, algumas estruturas corporais tornam-se comprometidas e, de fato, o expõe ao declínio da saúde e produtividade no trabalho. Daniel et al. ${ }^{1}$ atribuem que as principais causas de ausência no trabalho são decorrentes de doenças osteomusculares, transtornos mentais e traumatismos. Estima-se que entre 2011 e 2030 o impacto dos custos com assistência médica e improdutividade no trabalho será de U\$ 47 trilhões ${ }^{2}$.

Por outro lado, programas de exercício físico destinados a trabalhadores podem trazer grandes benefícios por promover qualidade de vida e melhoria das condições e modos de viver; expandir a potencialidade da saúde no âmbito coletivo e individual, diminuir as vulnerabilidades e riscos à saúde desinentes dos determinantes sociais, culturais, ambientais, econômicos e políticos ${ }^{3}$.

A prática de exercício físico necessita estar incorporada ao cotidiano das pessoas, na cultura popular, nos tratamentos médicos, na educação infantil e na organização familiar. Além disso, dados apontam que os custos com saúde individual e coletiva reduzem em populações fisicamente ativas ${ }^{4}$. Em um estudo foi observado que praticantes de ginástica laboral apresentaram redução dos níveis lipoproteína de baixa densidade (LDL), cortisol salivar e melhora da flexibilidade; quem realizou ginástica recreativa reduziu o LDL, circunferência da cintura e estresse percebido; e o grupo de relaxamento propiciou a diminuição da pressão, porcentagem de gordura, LDL, glicose e cortisol salivar ${ }^{5}$. Ramli et al. ${ }^{6}$ reportaram resultados positivos na gordura corporal, captação máxima de oxigênio e flexibilidade após a realização de um programa de saúde no local de trabalho. Esses achados apontam a importância da implantação de programas envolvendo atividade física para trabalhadores, visando a manutenção e melhora da saúde e qualidade de vida.

O Método Pilates é apontado como um exercício físico efetivo, capaz de propiciar condicionamento físico, flexibilidade, restauração postural, força, ampliação do controle motor, consciência e percepção corporal ${ }^{7}$. De acordo com a Associação Brasileira de Pilates ${ }^{8}$, Joseph Pilates atribuiu seis princípios ao seu método: concentração, respiração, controle, centralização de força, precisão e fluidez. Bellinate ${ }^{9}$ demonstrou que servidores que participaram de um programa do Método Pilates Solo e Bola, durante seis meses e frequência de duas vezes na semana, melhoraram os domínios da qualidade de vida, com escores mais significativos nos aspectos físicos e emocionais.

Em estudo com uma abordagem do método Mat Pilates, foi observado que o nível de sonolência mostrou melhora significativa $(\mathrm{p}=0,04)$ além da melhora da qualidade de vida $(\mathrm{p}<0,05)^{10}$. Cristóbal et al. ${ }^{11}$ reportaram que oito ou mais semanas de treino do método Pilates, com frequência de dois a quatro dias por semana, pode reduzir o percentual de gordura, dobras cutâneas e circunferências corporais. Gonçalves e $\mathrm{Lima}^{12}$, relataram que a maioria dos praticantes de Pilates obtiveram melhoria da qualidade de vida e saúde, e os que praticavam mais de um ano apresentaram maior capacidade funcional.

Portanto, o Método Pilates pode ser um grande instrumento de trabalho para com os servidores, pois se trata de uma modalidade com resultados benéficos de várias valências físicas. Assim, o presente estudo tem como pergunta de pesquisa: qual o efeito de uma abordagem de Mat Pilates na composição corporal, níveis de atividade física, qualidade de vida, flexibilidade e força em servidores?

Levando em conta a reduzida produção científica em estudos desenhados com intuito de analisar os benefícios do método Mat Pilates dentro do ambiente de trabalho, tendo como público alvo servidores de instituições de saúde. Sendo assim, essa abordagem é indispensável para ampliar o conhecimento, além de proporcionar novas perspectivas de incentivo e auxilio a saúde de trabalhadores, ampliando assim a produtividade das empreses. Desta forma, a pesquisa tem como objetivo avaliar o efeito de um programa Mat Pilates, sobre a composição corporal, qualidade de vida, nível de atividade física, flexibilidade e força em servidores de um hospital universitário.

\section{PROCEDIMENTOS METODOLÓGICOS}

Trata-se de um estudo quasi-experimental, do tipo pré- e pós-testes sem grupo controle. Como critérios de inclusão, os participantes deveriam ser servidores de uma instituição de saúde, de ambos os sexos e que não apresentassem limitações físicas para realização do programa de exercícios físicos. Foram excluídos os participantes que não atenderam a pelo menos $70 \%$ das aulas de Mat Pilates e aqueles que apresentaram qualquer contraindicação para com a realização das aulas e os que não responderam devidamente os questionários.

\section{Amostra e cuidados éticos}

Um total de 44 servidores do Hospital de Clínicas da Universidade Federal do Triângulo Mineiro (HC-UFTM), 
de ambos os sexos, e com idade entre 18 a 60 anos participaram desse estudo. $\mathrm{O}$ estudo foi divulgado por meio de cartazes afixados nos quadros de aviso do hospital.

Este estudo foi aprovado pelo Comitê de Ética em Pesquisa da Universidade Federal do Triângulo Mineiro, em 2018, sob o parecer $\mathrm{n}^{\mathrm{o}} 2.544 .358$. Os dados foram coletados após assinatura do Termo de Consentimento Livre Esclarecido pelos participantes.

\section{Procedimentos}

Após divulgação do estudo, os interessados fizeram inscrições online. A equipe de avaliação foi formada por profissionais de Educação Física e residentes multiprofissionais de Educação Física. Todas as avaliações foram realizadas no início e no final de 20 semanas de intervenção.

\section{Avaliação do nível de atividade física}

Para determinar o nível de atividade física foi aplicado o Questionário Internacional de Atividade Física (IPAQ) versão curta, sendo referido à semana anterior, apresentando perguntas em relação à frequência e duração da realização de atividades físicas vigorosas, moderadas e de caminhada. Os indivíduos foram classificados em muito ativo, ativo, irregularmente ativo ou sedentário ${ }^{13}$. Os servidores sedentários ou irregularmente ativos A e B foram classificados como inadequados, enquanto os ativos ou muito ativos foram classificados como adequados.

\section{Avaliação da qualidade de vida}

O questionário de avaliação de qualidade de vida e saúde (QVS-80) foi utilizado para determinar a qualidade de vida dos servidores. O instrumento possui 80 questões e é dividido em quatro domínios: saúde, atividade física, ambiente ocupacional e, percepção da qualidade de vida ${ }^{14}$.

\section{Avaliação antropométrica e da composição corporal}

A massa corporal foi medida por meio de uma balança digital (ActlifeSlimpro ${ }^{\circledR}$, MODELO, PAÍS) com capacidade para $180 \mathrm{~kg}$. Estatura foi medida com uso de estadiômetro de alumínio (Sanny®, Brasil). Em seguida, o índice de massa corporal (IMC) foi calculado (massa corporal em $\left.\mathrm{kg} / \mathrm{estatura} \mathrm{em} \mathrm{m}^{2}\right)^{15}$.

Perímetros do pescoço, cintura, abdômen e quadril, foram realizados com uso de trena antropométrica de 2 metros (Cescorf ${ }^{\circledR}$, Porto Alegre - Brasil).
A composição corporal é entendida como a dimensão entre os diferentes componentes corporais e a massa corporal total, sendo constituída pela percentagem de gordura corporal e massa corporal magra ${ }^{16}$. Medidas das pregas de adiposidade subcutâneas subescapular, peitoral, axilarmedia, suprailíaca, abdominal, bicipital e coxa foram obtidas com uso de adipômetro (TBW Lange ${ }^{\circledR}$, Skinfold Caliper-MI, EUA) de acordo com a técnica de Moreira ${ }^{17}$. Para a densidade corporal foi utilizada a equação de $\sum 7^{18}$. Os valores de percentual de gordura e de massa livre de gordura foram preditos, respectivamente, pelas equações de Siri $^{19}$ e Goldman e Becklake ${ }^{20}$.

\section{Teste de força abdominal}

O teste consiste na execução de flexões do tronco, partindo da posição de costas e pés apoiados no solo, devendo cotovelos tocar nos joelhos. A contabilização do número máximo de flexões realizadas durante 60 segundos foi classificada como resistência muscular abdominal fraca, abaixo da média, média, acima da média e excelente ${ }^{21}$. Em seguida, foram categorizadas como adequado as classificações fraca, abaixo da média e como inadequado as classificações média, acima da média e excelente.

\section{Teste de flexibilidade}

O teste de flexibilidade consiste na flexão de tronco, na posição sentada, utilizando o banco de Wells (DA marca Danny ${ }^{\circledR}$, PAÍS). O participante avaliado teve que flexionar o tronco sobre o quadril, empurrando o suporte de acrílico na régua milimétrica. $O$ teste foi realizado três vezes, considerando-se a maior distância atingida, seguindo a padronização do Canadian Standardized Test of Fitness ${ }^{22}$. Foram classificados como inadequado os participantes que tiveram como resultado da flexibilidade ruim e abaixo da média, e adequado com média, acima da média e excelente.

\section{Intervenção}

Todas atividades foram realizadas em uma sala ampla e ventilada na Universidade Federal do Triângulo Mineiro. A intervenção teve duração de 20 semanas, sendo realizada uma sessão por semana com duração média de uma hora. As sessões foram realizados no solo (mat), sendo elas divididas em aquecimento com três exercícios voltados para o alongamento e aquecimento de articulações 
a serem trabalhadas na sessão; parte principal com dez exercícios com uma série com 15 repetições por exercício, utilizando materiais como therabans, bola de leite, bola suíça e halteres, não havendo intervalo para descanso; e volta a calma com três exercícios de alongamento e relaxamento. Todos movimentos eram executados durante a expiração.

\section{Análise dos dados / estatística}

Após a obtenção e tabulação dos dados, os mesmos foram analisados por meio do programa estatístico Statistical Package for the Social Sciences (SPSS), versão 21.0. Inicialmente foi realizada a estatística descritiva para obtenção de valores de tendência central e dispersão, em seguida foi aplicado o Teste t pareado a fim de analisar as variáveis contínuas. Posteriormente foi aplicado o teste de correlação de Pearson para medir o grau de relação linear entre duas variáveis. Por fim foi realizado o teste de quiquadrado de Pearson para as variáveis categóricas. O nível de significância adotado foi de $\mathrm{p}<0,05$.

\section{RESULTADOS}

A amostra obtida foi composta de 44 participantes. Foram excluídos 22 participantes por preenchimento incorreto dos instrumentos de pesquisa ou por não atingirem o mínimo de aulas pré-determinadas, perfazendo uma amostra final de 22 servidores. Foi identificada maior proporção de mulheres $(\mathrm{n}=19,86,36 \%)$; com renda familiar mensal de $\mathrm{R} \$ 927,00$ a $\mathrm{R} \$ 1669,00(\mathrm{n}=1,4,55 \%)$, de $\mathrm{R} \$ 1669,00$ a $\mathrm{R} \$ 2804,00(\mathrm{n}=1,4,55 \%)$, de $\mathrm{R} \$ 2804,00$ a $\mathrm{R} \$ 4648,00(\mathrm{n}=13,59,09 \%)$, e acima de $\mathrm{R} \$ 4648,00$ $(\mathrm{n}=7,31,82 \%)$; com doenças recorrentes $(\mathrm{n}=4,18,18 \%)$ com hipertensão arterial sistêmica, $(\mathrm{n}=3,13,64 \%)$ com colesterol alto, $(\mathrm{n}=2,9,09 \%)$ com depressão, $(\mathrm{n}=1$, $4,55 \%)$ com doença da tireóide, ( $\mathrm{n}=1,4,55 \%)$ com triglicerídeos alto, $(\mathrm{n}=1,4,55 \%)$ com doença respiratória; com dores e desconfortos em coluna $(\mathrm{n}=9,40,91 \%)$, pernas e pés $(\mathrm{n}=8,36,36 \%)$, cabeça e olhos $(\mathrm{n}=4$, $18,18 \%)$, braços e ombros $(\mathrm{n}=3,13,64 \%)$, e punhos e mãos $(n=3,13,64 \%)$. Outras características da amostra e a comparação pré e pós intervenção podem ser elucidadas na Tabela 1. Houve redução significativa $(p<0,05)$ do perímetro da cintura, pressão arterial sistólica e diastólica, massa corporal gorda, percentagem de gordura, melhora dos domínios percepção da qualidade de vida, saúde e média da qualidade de vida.
Tabela 1 - Perfil antropométrico, hemodinâmico, composição corporal e qualidade de vida de servidores públicos antes e após intervenção de 20 semanas de Mat Pilates $(\mathrm{n}=22)$.

\begin{tabular}{|c|c|c|c|}
\hline & \multicolumn{2}{|c|}{ Média \pm desvio padrão } & \multirow[t]{2}{*}{ p-valor } \\
\hline & Pré & Pós & \\
\hline \multicolumn{4}{|l|}{ Antropometria } \\
\hline Massa corporal (kg) & $74,30 \pm 16,95$ & $73,90 \pm 15,82$ & 0,387 \\
\hline Estatura (m) & $1,63 \pm 0,12$ & $1,63 \pm 0,12$ & - \\
\hline $\begin{array}{l}\text { Índice de Massa } \\
\text { Corporal }\left(\mathrm{kg} / \mathrm{m}^{2}\right)\end{array}$ & $27,71 \pm 5,70$ & $27,62 \pm 5,69$ & 0,548 \\
\hline $\begin{array}{l}\text { Perímetro do } \\
\text { Pescoço }(\mathrm{cm})\end{array}$ & $34,54 \pm 4,14$ & $34,27 \pm 3,87$ & 0,050 \\
\hline $\begin{array}{l}\text { Perímetro da } \\
\text { Cintura }(\mathrm{cm})\end{array}$ & $84,15 \pm 12,79$ & $82,86 \pm 13,01$ & $0,038^{*}$ \\
\hline $\begin{array}{l}\text { Perímetro do } \\
\text { Abdômen }(\mathrm{cm})\end{array}$ & $94,09 \pm 12,25$ & $93,41 \pm 12,10$ & 0,312 \\
\hline $\begin{array}{l}\text { Perímetro do } \\
\text { Quadril (cm) }\end{array}$ & $105,72 \pm 9,37$ & $105,57 \pm 8,69$ & 0,845 \\
\hline $\begin{array}{l}\text { Perímetro do } \\
\text { Braço }(\mathrm{cm})\end{array}$ & $31,40 \pm 4,51$ & $31,65 \pm 4,32$ & 0,550 \\
\hline \multicolumn{4}{|l|}{ Hemodinâmica } \\
\hline $\begin{array}{l}\text { Pressão Arterial } \\
\text { Sistólica (mmHg) }\end{array}$ & $121,36 \pm 13,56$ & $112,73 \pm 14,53$ & $0,001 *$ \\
\hline $\begin{array}{l}\text { Pressão Arterial } \\
\text { Diastólica (mmHg) }\end{array}$ & $78,64 \pm 11,67$ & $70,91 \pm 10,19$ & $0,001 *$ \\
\hline $\begin{array}{l}\text { Frequência } \\
\text { Cardíaca }(\mathrm{bpm})\end{array}$ & $71,27 \pm 9,35$ & $72,18 \pm 9,17$ & 0,568 \\
\hline \multicolumn{4}{|l|}{ Composição corporal } \\
\hline $\begin{array}{l}\text { Massa Corporal } \\
\text { Gorda }(\mathrm{kg})\end{array}$ & $21,97 \pm 8,23$ & $20,85 \pm 7,87$ & $0,008^{*}$ \\
\hline $\begin{array}{l}\text { Massa Corporal } \\
\text { Magra }(\mathrm{kg})\end{array}$ & $52,33 \pm 13,41$ & $52,98 \pm 12,72$ & 0,058 \\
\hline $\begin{array}{l}\text { Percentual de Gordura } \\
\text { Corporal }\end{array}$ & $29,21 \pm 7,93$ & $27,90 \pm 7,69$ & $0,003 *$ \\
\hline \multicolumn{4}{|l|}{ Qualidade de vida } \\
\hline Saúde & $68,88 \pm 22,70$ & $81,87 \pm 7,41$ & $0,018^{*}$ \\
\hline Atividade Física & $54,55 \pm 15,93$ & $50,18 \pm 9,59$ & 0,249 \\
\hline $\begin{array}{l}\text { Ambiente } \\
\text { Ocupacional }\end{array}$ & $57,10 \pm 11,51$ & $62,23 \pm 10,21$ & 0,067 \\
\hline $\begin{array}{l}\text { Percepção da } \\
\text { Qualidade de Vida }\end{array}$ & $64,35 \pm 15,39$ & $74,10 \pm 8,28$ & $0,017^{*}$ \\
\hline $\begin{array}{l}\text { Média da Qualidade } \\
\text { de Vida }\end{array}$ & $61,22 \pm 8,84$ & $67,10 \pm 6,06$ & $0,011^{*}$ \\
\hline
\end{tabular}

$* \mathrm{p}<0,05$.

A análise do delta das variáveis apontou correlação significativa positiva entre frequência cardíaca e IMC, perímetro do quadril e massa corporal gorda; do perímetro do abdômen com a massa livre de gordura; perímetro do quadril com a massa corporal gorda (Tabela 2). 
Feldner CB, et al. Efeitos de um programa de Mat Pilates sobre indicadores de saúde. Rev Ter Ocup Univ São Paulo. 2020 set./dez.;30(3):160-6.

Tabela 2 - Correlação entre valores delta de variáveis antropométricas, hemodinâmicas, composição corporal e qualidade de vida de servidores públicos antes e após intervenção de 20 semanas de Mat Pilates $(\mathrm{n}=22)$.

\begin{tabular}{|c|c|c|c|c|c|c|c|c|c|c|c|}
\hline & $\Delta \mathrm{IMC}$ & $\Delta P A S$ & DPAD & $\Delta F C$ & $\Delta \mathbf{P P}$ & $\Delta P C$ & $\mathbf{\Delta P A}$ & $\Delta P Q$ & $\triangle M C G$ & $\Delta M L G$ & $\Delta \mathrm{MQV}$ \\
\hline$\Delta \mathrm{MC}$ & ,988** &,- 125 &, 056 &, $516^{*}$ & ,623** &, $425^{*}$ &, $724^{* *}$ &, $655^{* *}$ & ,694** &, $602^{* *}$ &,- 065 \\
\hline$\Delta \mathrm{IMC}$ & &,- 130 & ,046 &, $527^{*}$ &, $621^{* *}$ & ,415 &, $736^{* *}$ &, $653^{* *}$ &, $680^{* *}$ &, $603^{* *}$ &,- 038 \\
\hline$\triangle \mathrm{PAS}$ & & & ,416 &,- 115 &,- 150 &,- 073 &,- 251 &,- 172 &,- 135 &,- 013 & ,295 \\
\hline$\triangle \mathrm{PAD}$ & & & &,- 128 &,- 236 & ,101 &, 111 &,- 066 &,- 078 & ,242 & ,090 \\
\hline$\Delta \mathrm{FC}$ & & & & & ,393 &, 344 & ,372 &, $469^{*}$ &, $566^{* *}$ &, 031 &,- 136 \\
\hline$\Delta \mathrm{PP}$ & & & & & &,- 062 & ,306 & ,383 & ,414 & ,403 &,- 359 \\
\hline$\Delta \mathrm{PC}$ & & & & & & &, $614^{* *}$ & ,294 & ,215 &, 373 &,- 109 \\
\hline$\Delta \mathrm{PA}$ & & & & & & & &, $665^{* *}$ & ,407 &, $582^{* *}$ & ,166 \\
\hline$\Delta \mathrm{PQ}$ & & & & & & & & &, $712^{* *}$ &, 132 & ,168 \\
\hline$\triangle \mathrm{MCG}$ & & & & & & & & & &,- 139 &,- 034 \\
\hline$\Delta \mathrm{MLG}$ & & & & & & & & & & &,- 046 \\
\hline
\end{tabular}

Legenda: Massa Corporal (MC), Índice de Massa Corporal (IMC), Pressão Arterial Sistólica (PAS), Pressão Arterial Diastólica (PAD), Frequência (FC), Perímetro do Pescoço (PP), Perímetro da Cintura (PC), Perímetro do Abdômen (PA), Perímetro do Quadril (PQ), Massa Corporal Gorda (MCG), Massa Livre de Gordura (MLG), Média da Qualidade de Vida (MQV). ** denota correlação significativa ao nível 0,01 (2 extremidades). * denota correlação significativa no nível 0,05 (2 extremidades).

Houve aumento $(\mathrm{p}<0,05)$ na proporção de participantes a alcançar adequação dos níveis de atividade física, força abdominal e flexibilidade após 20 semanas de intervenção (Tabela 3).

Tabela 3 - Qui-quadrado de Pearson entre variáveis de nível de atividade física, força abdominal e flexibilidade de servidores públicos antes e após intervenção de 20 semanas de Mat Pilates $(n=22)$.

\begin{tabular}{lccc}
\hline & Pré & Pós & P-valor \\
\hline Nível de Atividade Física & & & \\
Inadequado & $54,5 \%$ & $36,4 \%$ & $0,019^{*}$ \\
Adequado & $45,5 \%$ & $63,6 \%$ & \\
Força Abdominal & & & \\
Inadequado & $68,2 \%$ & $40,9 \%$ & $0,008^{*}$ \\
Adequado & $31,8 \%$ & $59,1 \%$ & \\
Flexibilidade & & & \\
Inadequado & $81,8 \%$ & $68,2 \%$ & $0,001^{*}$ \\
Adequado & $18,2 \%$ & $31,8 \%$ & \\
\hline
\end{tabular}

$* \mathrm{p}<0,05$.

\section{DISCUSSÃO}

O presente estudo teve como proposta verificar e analisar os efeitos de um programa de Mat Pilates, uma vez por semana, nos parâmetros de saúde em servidores de um hospital universitário. Como principais achados destacase a redução da adiposidade corporal, pressão arterial e melhora do nível de atividade física, força abdominal, flexibilidade e qualidade de vida dos participantes da intervenção. Resultados similares foram encontrados por Martins-Meneses et al. ${ }^{23}$, que utilizaram intervenção de Mat Pilates, duas vezes na semana, durante 16 semanas em um grupo de mulheres hipertensas medicadas.

Várias são as barreiras encontradas por servidores públicos de hospitais universitários para a não adoção de um estilo de vida saudável durante horário de trabalho. Destacam-se a falta de programas voltados para esse fim; falta de espaços adequados para prática de atividade física; natureza imprevisível da atividade que compromete assiduidade aos programas criados; enfim, o planejamento de ações orientados a melhorar parametros de saúde nesses profissionais devem levar em conta essas questões para alcance de sucesso.

A redução da adiposidade na regiao central do corpo verificada no presente estudo tem sido associada a redução do risco de doenças cardiovasculares. Embora não tenhamos utilizado métodos de imagem, encontramos redução do perímetro de cintura para valores próximos ao ponto de corte $(\geq 80 \mathrm{~cm}$ para mulheres e $\geq 94 \mathrm{~cm}$ para homens) segundo a Associação Brasileira para o Estudo da Obesidade e da Síndrome Metabólica ${ }^{24}$. Consequentemente, alterações positivas foram também encontradas na massa corporal gorda e percentual de gordura. Em revisão de literatura, Cristóbal et al. ${ }^{11}$, reportaram que o treinamento do método Pilates, com frequência de dois a quatro dias por semana, mais de 
8 semanas, reduz o percentual de gordura, dobras cutâneas e circunferências.

Este estudo observou melhoras na qualidade de vida nos domínios da saúde, percepção da qualidade de vida e a média da mesma. Foi notado melhora da qualidade de vida nos domínios emocionais e físicos, após a aplicação de um protocolo de três meses, duas vezes na semana, do método Mat Pilates em voluntários sedentários ${ }^{10}$. Além disso, Gonçalves e Lima ${ }^{12}$, obtiveram melhoria da qualidade de vida e saúde de praticantes do método Pilates.

\section{CONCLUSÃO}

A implantação de projetos visando a saúde dos trabalhadores se mostra indispensável e de extrema importância, pois aumentando a disposição e o nível de qualidade de vida do trabalhador, melhor será seu desempenho na empresa. O programa de Mat Pilates se mostra eficiente para ampliar a saúde de servidores, visto que um simples treinamento semanal proporcionou melhoras significativas em parâmetros hemodinâmicos, físicos e de qualidade de vida.

Contribuiçães dos autores: Feldner CB, colaborou em todas etapas de produção do artigo (concepção, coleta, organização e análise dos dados, redação e revisão). Cezário NF, auxiliou na coleta e revisão do artigo. Mendes EL, contribuiu na análise dos dados e revisão crítica do artigo. Todos autores aprovaram a versão final do texto.

\section{REFERÊNCIAS}

1. Daniel E, Koerich CRC, Lang A. O perfil do absenteísmo dos servidores da prefeitura municipal de Curitiba, de 2010 a 2015. Rev Bras Med Trab. 2017; 15(2):142-9. http://dx.doi. br/10.5327/Z1679443520176021.

2. Bloom DE, Cafiero ET, Jané-Llopis E, Abrahams-Gessel S, Bloom LR, Fathima S, et al. The Global Economic Burden of Noncommunicable Diseases. Geneva: World Economic Forum; 2011 [cited 2019 Jan 10]. Available from: http://www3.weforum.org/docs/WEF_Harvard_HE_ GlobalEconomicBurdenNonCommunicableDiseases_2011.pdf

3. Brasil. Ministério da Saúde. Portaria no 2446, de 11 de novembro de 2014. Redefine a Política Nacional de Promoção da Saúde (PNPS). Diário Oficial da União, 2014, nov. 13.

4. Araújo DSMS, Araújo CGS. Aptidão física, saúde e qualidade de vida relacionada à saúde em adultos. Rev Bras Med Esporte. 2000;6(5):194-203. http://dx.doi.org/10.1590/ S1517-86922000000500005

5. Osiecki ACV. Efeitos das práticas de ginásticas recreativa, laboral e relaxamento sobre os fatores de risco cardiovasculares, estresse e qualidade de vida em servidores [Tese]. Curitiba: Universidade Federal do Paraná; 2013 [citado 12 jan. 2019]. Disponível em: https://acervodigital. ufpr.br/bitstream/handle/1884/36150/R\%20-\%20T\%20 -\%20ANA\%20CLAUDIA\%20VECCHI\%20OSIECKI. pdf? sequence $=1 \&$ isAllowed $=y$

6. Ramli A, Henry LJ, Liang YF, Beh Jy. Effects of a worksite health programme on the improvement of physical health among overweight and obese civil servants: a pilot study.
Malays J Med Sci. 2013;20(5):54-60. Available from: https:// www.ncbi.nlm.nih.gov/pmc/articles/PMC3957360/

7. Blum, C.L. Chiropractic and Pilates therapy for thetreatment of adults coliosis. J Manipulative Physiol Ther. 2002; 25(4):E3. http://dx.doi.org/10.1067/mmt.2002.123336

8. Associação Brasileira de Pilates. Original Pilates [acesso 28 2018]. Disponível em: http://www.abpilates.com.br/site/ metodos.asp.

9. Bellinate MC. A repercussão do método pilates solo e bola na qualidade de vida dos servidores da universidade federal de mato grosso do sul - Campus Campo Grande [monografia]. Campo Grande: Universidade Católica Dom Bosco; 2015 [citado 15 ago. 2018]. Disponível em: http://site.ucdb.br/public/md-dissertacoes/19992-final.pdf

10. Leopoldino AAO, Avelar NCP, Passos GB, Santana NTP, Teixeira VP, de Lima VP, et al. Effect of Pilates on sleep quality and quality of life of sedentary population. $\mathrm{J}$ Bodywork Mov. 2013;17(1):5-10. https://doi.org/10.1016/j. jbmt.2012.10.001

11. Cristóbal RV, Alacid F, Esparza-Ros F, Muyor JM, LópezMinarro PA. Pilates: efecto sobre lacomposición corporal y lasvariables antropométricas. Apunts Med Esport. 2014; 49(183):85-91. https://doi.org/10.1016/j.apunts.2013.12.002

12. Gonçalves OS, Lima PO. Percepção de saúde e qualidade de vida: um inquérito com praticantes de pilates. Rev Fisioter $\mathrm{S}$ Fun. 2014;3(1):1-17. Disponível em: http://www.repositorio. ufc.br/ri/bitstream/riufc/9808/1/2014_art_poplima.pdf 
13. Matsudo SM, Araújo T, Matsudo V, Andrade D, Andrade E, Oliveira LC, et al. Questionário Internacional de Atividade Física (IPAQ): estudo de validade e reprodutibilidade no Brasil. Rev Bras Ativ Fís Saúde. 2001; 6(2):5-18. https://doi. org/10.12820/rbafs.v.6n2p5-18

14. Mendes RA, Leite N. Ginástica laboral: princípios e aplicações práticas. Barueri (SP): Manole; 2008. Cap. 3. 2008.

15. Thomas AE, Mc Kay DA, Cutlip MB. A monograph method for assessing body weight. Am J Clin Nutr. 1976; 29:302-4, 1976.

16. Mourão PJM, Gonçalves FJM. A avaliação da composição corporal: a medição de pregas adiposas com técnica para a avaliação da composição corporal. Motri. 2008;4(4):13-21. Disponível em: http://www. scielo.mec.pt/scielo.php?script=sci_arttext\&pid=S1646107X2008000400003\&lng=es

17. Moreira MH. Avaliação das pregas adiposas. Prova de aptidão. Universidade de Trás-os-Montes e Alto Douro: Vila Real; 1995.

18. Jackson AS, Pollock ML. Generalized equations for predicting body density of men. Br J Nutr. 1978;40(3):497-504.

19. Siri WE. Body composition from fluid spaces and density. In: Brozek J, Henschel A, editors. Techniques for measuring body composition. Washington DC: National Academy of Sience; 1961. p.223-44.

20. Goldman HI, Becklake MR. Respiratory function tests: normal values of medium altitudes and the prediction of normal results. Am Rev Respir Dis. 1959;79(4):457-67.

21. Pollock ML, Wilmore JH. Medicina e esporte. In: exercícios na saúde e na doença: avaliação e prescrição para prevenção e reabilitação. $2^{\mathrm{a}}$ ed. Rio de Janeiro; 1993. p.125-7.

22. Canadian Standardized Test of Fitness. Operations manual. Fitness and amateur sport. 3rd ed. Ottawa: Minister of State; 1986.

23. Martins-Meneses DT, Antunes HKM, de Oliveira NRC, Medeiros A. Mat Pilates training reduced clinical and ambulatory blood pressure in hypertensive women using antihypertensive medications. Int J Cardiol. 2015;179:262-8. https://doi.org/10.1016/j.ijcard.2014.11.064

24. Associação Brasileira para o Estudo da Obesidade e da Síndrome Metabólica (ABESO). Diretrizes Brasileiras de Obesidade 2016. 4ª . ed. São Paulo; 2016 [citado 27 mar. 2019]. Disponível em: http://www.abeso.org.br/uploads/ downloads/92/57fccc403e5da.pdf 